\title{
Strongly nonlinear wave dynamics in a chain of polymer coated beads
}

\author{
C. Daraio ${ }^{1}$ and V. F. Nesterenko ${ }^{1,2, *}$ \\ ${ }^{1}$ Materials Science and Engineering Program, University of California at San Diego, La Jolla, California 92093-0418, USA \\ ${ }^{2}$ Mechanical and Aerospace Engineering Department, University of California at San Diego, La Jolla, California 92093-0411, USA
}

(Received 3 October 2005; published 21 February 2006)

\begin{abstract}
Strongly nonlinear phononic crystals were assembled from a chain of Parylene- $\mathrm{C}$ coated steel spheres in a polytetrafluoroethylene holder. This system exhibits strongly nonlinear properties and extends the range of materials supporting sonic-vacuum-type behavior. The combination of a high density core and a soft (low elastic modulus) coating ensures a relatively low velocity of wave propagation. The bead contact interaction caused by the deformation of the Parylene coating can be described by classical nonlinear elastic Hertz theory with an effective value of the elastic modulus equal to $15 \mathrm{GPa}$ for the contact interaction. Strongly nonlinear solitary waves excited by impacts were investigated experimentally and compared to chains composed of uniform steel beads. Fracture of the polymer coating was detected under relatively large pulse amplitude.
\end{abstract}

DOI: 10.1103/PhysRevE.73.026612

PACS number(s): 05.45.Yv, 46.40.Cd, 43.25. $+\mathrm{y}, 45.70 .-\mathrm{n}$

\section{INTRODUCTION}

In recent years the interest in understanding the dynamic behavior of granular materials has known a substantial increase [1-34]. Strongly nonlinear one-dimensional chains of beads of different materials represent the simplest examples of media important for the development of a different wave dynamics.

The strongly nonlinear behavior of granular matter arises from its inherent "double nonlinearity" due to the Hertzian contact interaction [35] between granules under compression and the zero interaction force under tensile stresses. Such behavior appears only if the granular material is "weakly" compressed. If the initial precompressive force is zero, such a system cannot support classical phonons and for these reasons was called a "sonic vacuum" [5]. The particle contact interaction under "strong" precompression alters the wave propagation behavior from the strongly nonlinear type to the linear or weakly nonlinear Korteweg-de Vries type [8]. A property deriving from this "tunability" of the contact interaction is the ability to control the wave propagation regime simply by adjusting the amount of precompression acting on the system [33] or by varying the elastic properties at the particle contacts or the mass of the particles.

Coating a high density particle such as the steel beads considered in this study with a soft polymeric layer might ensure a better control of impulse transformation and propagation characteristics, allowing applicability in a large variety of engineering solutions. The results obtained from these analyses may lead to applications in sound focusing devices (acoustic lenses), very short transmission delay lines, sound and shock absorption layers, and sound scramblers. Granular beds composed from iron particles are used for designing systems able to transform impacts from heavy strikers and contact explosion [14]. The soft coating is important for delaying and reducing the amplitude and wave speed in the medium, adjusting, for example, the protective granular bar-

*Electronic address: vnesterenko@ucsd.edu rier to a wider spectrum of dynamic loads. Parylene-C is a very versatile viscoelastic material, widely biocompatible, with a very low friction coefficient, and low elastic modulus. Its properties, combined with the wide commercial availability of Parylene-C thin film coating, allowed it to be suitable for the assembling of strongly nonlinear systems. To the best of our knowledge no other results on the pulse propagation in chains composed of coated particles were previously published.

\section{THEORETICAL ANALYSIS}

For the theoretical treatment, we consider that the particle's stainless steel core with radius $R^{\prime}$ behaves as a rigid body and the depth of the deformed contact zone is smaller than the thickness of the coated layer $\Delta$. In the initial conditions the beads have all point contacts with their neighboring particles and the particle interaction is limited only to the deformation of their coating. Finally we assume that the time of the particle interaction is significantly longer than the time of the wave propagation inside the particle, ensuring the application of a quasistatic interaction law with constant value of elastic modulus. Under these assumptions, using the Hertzian expression of force between the granules [35],

$$
F=\frac{2 E}{3\left(1-\nu^{2}\right)}\left(\frac{R^{\prime}+\Delta}{2}\right)^{1 / 2}\left[2\left(R^{\prime}+\Delta\right)-\left(x_{2}-x_{1}\right)\right]^{3 / 2}
$$

we obtain the following equation of motion for a onedimensional chain of identical uniform coated beads (Fig. 1) similar to the one presented in [14]:

$$
\ddot{u}_{i}=A\left(\delta_{0}-u_{i}+u_{i-1}\right)^{3 / 2}-A\left(\delta_{0}-u_{i+1}+u_{i}\right)^{3 / 2},
$$

where

$$
A=\frac{E a^{1 / 2}}{3\left(1-\nu^{2}\right) M}, \quad a=2\left(R^{\prime}+\Delta\right)
$$

and 


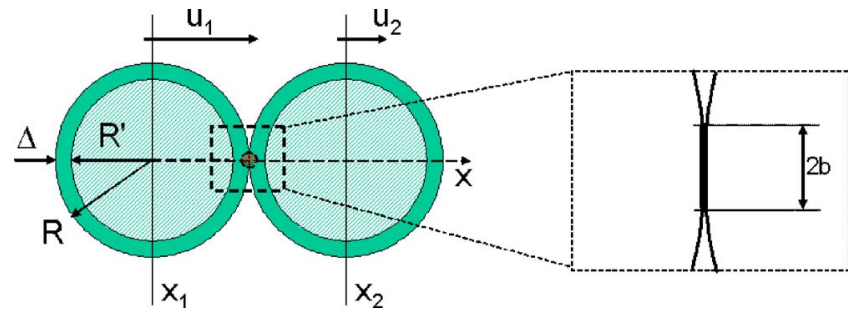

FIG. 1. (Color online) Schematic diagram showing the contact interaction area between two Parylene coated particles. The inset identifies an enlargement of the contact interaction area.

$$
M=m_{\mathrm{ss}}+m_{p}=\frac{4}{3} \pi R^{\prime 3} \rho_{\mathrm{ss}}+\frac{4}{3} \pi\left[\left(R^{\prime}+\Delta\right)^{3}-R^{\prime 3}\right] \rho_{p} .
$$

Here $\delta_{0}$ is the initial displacement between particles due to the static precompression, $E$ is the elastic modulus, $\nu$ is the Poisson coefficient of Parylene-C, and $\Delta$ represents the Parylene coating thickness. The diameter of the particles is $a$, the mass $M\left(m_{\mathrm{ss}}\right.$ is the mass of the stainless steel core and $m_{p}$ is the mass of the coating) and the displacement of the $i$ th particle is $u_{i}$.

When a weak precompression is applied to the system the long wave equation for the particle displacement $u$ is

$$
u_{\mathrm{tt}}=-c^{2}\left(\left(-u_{x}\right)^{3 / 2}+\frac{a^{2}}{10}\left\{\left(-u_{x}\right)^{1 / 4}\left[\left(-u_{x}\right)^{5 / 4}\right]_{x x}\right\}\right)_{x}
$$

where

$$
\begin{aligned}
& -u_{x}>0, \quad c^{2}=\frac{E a^{3}}{3\left(1-\nu^{2}\right) M}, \\
& \text { and the sound speed } c_{0}=\left(\frac{3}{2}\right)^{1 / 2} c \xi_{0}^{1 / 4} .
\end{aligned}
$$

Here $\xi_{0}$ is the initial strain in the system. It should be noticed that $c$ does not represent the sound speed in the chain, while $c_{0}$ corresponds to the long wave sound speed at initial strain $\xi_{0}$ in the system. Equation (3) for high amplitude pulses (or for negligible precompression) has no characteristic wave speed. In this case, despite their complex nature, Eqs. (3) and (2) support supersonic solitary waves with finite length equal to only five particle diameters [14].

In the leading approximation, the force $F_{m}$ between particles in a discrete chain is related to the maximum strain $\xi_{m}$ in the solitary wave as follows:

$$
F_{m}=\frac{4 E}{3\left(1-\nu^{2}\right)}\left(R^{\prime}+\Delta\right)^{2} \xi_{m}^{3 / 2}
$$

Also, from [14] we know that for a solitary wave in sonic vacuum the speed is equal:

$$
V_{s}=\frac{2}{\sqrt{5}} c \xi_{m}^{1 / 4}
$$

Combining Eqs. (4) and (5) we obtain the relation between the solitary wave speed and the maximum force between particles:

$$
V_{s}=\frac{2}{\sqrt{5}}\left(\frac{E a^{7 / 2}}{3\left(1-\nu^{2}\right)}\right)^{1 / 3} \frac{F_{m}^{1 / 6}}{\sqrt{M}} .
$$

It is evident that the speed of this wave has a nonlinear dependence on the maximum strain $\xi_{m}$ or force $F_{m}$ and can be infinitesimally small if the amplitude of the wave is small. For chains composed of uniform beads of different materials the existence of this solitary wave was verified in numerical calculations and in experiments $[1-3,6,10]$.

The addition of significant initial precompression on the system is known to modify the wave propagation behavior, for example due to the presence of gravitational precompression in the vertical alignment of the chains or due to the added prestress by another external source. The speed of the solitary wave $V_{s}$ in a chain of coated beads with applied static precompressive force $\left(F_{0}\right)$ is derived in a similar way as in [28] for uniform particles:

$$
V_{s}=\left(\frac{2}{15}\right)^{1 / 2}\left(\frac{3 E^{2} F_{0} a^{7}}{M^{3}\left(1-\nu^{2}\right)^{2}}\right)^{1 / 6} \frac{1}{f_{r}^{2 / 3}-1}\left(3+2 f_{r}^{5 / 3}-5 f_{r}^{2 / 3}\right)^{1 / 2} \text {. }
$$

The speed $V_{s}$ has a nonlinear dependence on the maximum force $f_{r}=F_{m} / F_{0}$ but can be considered approximately constant at any relatively narrow interval of its amplitude due to the small exponent present in the power law. Equation (6) can be obtained as a limit of Eq. (7) when the precompressive force $\left(F_{0}\right)$ is approaching zero and the equation for the sound speed $c_{0}$ can be recovered when $f_{r}$ is close to 1 .

The force dependence on displacement may still obey an equation similar to Hertzian theory [Eq. (1)] even if the depth of the deformed area is comparable to or larger than the thickness of the coating. In this case the elastic modulus $E$ in Eq. (7) represents an effective elastic modulus for the contact interaction of the composite particles and can be different from the elastic modulus of the coating.

If the dependence of the contact force on displacement between composite particles can be approximated by a general power law with exponent different from the Hertzian value 1.5, an equation similar to Eq. (7) can be derived with different exponents and coefficients based on Eq. 1.127 in [14]. It should be mentioned that the width of the solitary wave is related to the value of the exponent in the power law dependence of the contact force on displacement. The properties of strongly nonlinear solitary waves were used to identify the contact interaction law between beads made from different materials [10].

\section{EXPERIMENTAL SETUP}

We assembled strongly nonlinear chains from steel bearing balls (diameters of 4.76 and $2.38 \mathrm{~mm}$ ) coated with a $50-\mu \mathrm{m}$-thick layer of Parylene-C polymer (AcraBall Inc.) via vaporization processes [Fig. 2(a)]. The properties of Parylene-C provided by the manufacturer are the following: Young modulus $E=2760 \mathrm{MPa}$, density $=1289 \mathrm{~kg} / \mathrm{m}^{3}$, Poisson coefficient $\nu=0.388$ [36]. The yield strength $(Y)$ of thin films of Parylene- $\mathrm{C}$ is reported to be at $59 \mathrm{MPa}$ [37].

Parlylene-C is a polymeric viscoelastic material with a low elastic modulus. It is widely used in different areas from 

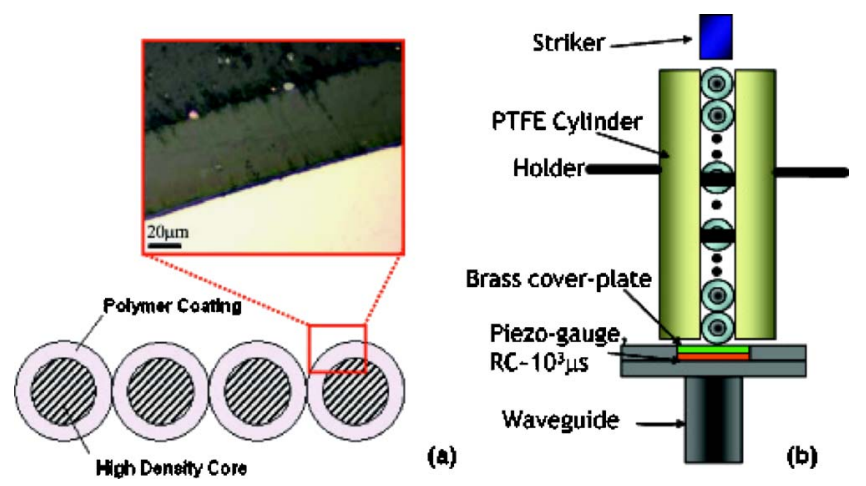

FIG. 2. (Color online) Experimental setup for testing of onedimensional strongly nonlinear chains. (a) Schematic diagram of the chain and optical micrograph showing the Parylene-C polymer coating. (b) Setup used for single chain testing, showing the particles with embedded sensors shown by a black central bar.

electronic to biomedical applications [37]. Its properties can be very attractive to ensure a low speed of soliton propagation and a good tunability of the system. At the same time it is not evident that a chain formed from this type of composite beads would support strongly nonlinear solitary waves based on Hertzian interaction, such as for example chains made from uniform linear elastic materials like steel $[14,6,32]$. In particular, the role of dissipation and deviation from the linear elastic law under high strain rate deformation (about $10^{2}-10^{3} \mathrm{~s}^{-1}$ ) can influence the contact interaction and the wave dynamics. Additionally, the geometrical constraints introduced by the stiffer steel core and the interfacial phenomena between the Parylene-C coating and the steel core, for example due to the weak adhesion, may contribute to the contact deformation and dissipation and affect the dynamic behavior of the chains. Chains of beads made from nylon [10] with elastic modulus 23\% larger than Parylene-C and chains made of polytetrafluoroethylene (PTFE) [28] with a nominal elastic modulus $\sim 5$ times smaller than Parylene-C demonstrated the validity of the Hertz-type interaction law and supported propagation of strongly nonlinear solitary waves.

One-dimensional chains were assembled from 21 Parylene-C coated steel beads (with diameter $a=4.86 \mathrm{~mm}$ and mass $0.44 \mathrm{~g}$ ) vertically aligned in a PTFE holder with inner diameter $5 \mathrm{~mm}$ [Fig. 2(b)]. Pulses of different durations and amplitudes were generated by impacts of alumina $\left(\mathrm{Al}_{2} \mathrm{O}_{3}\right)$ cylinders (with masses 0.47 and $1.23 \mathrm{~g}$ ) on the top particle of the chain from different heights.

In the described setup we included three calibrated piezo sensors $\left(R C \sim 10^{3} \mu \mathrm{s}\right)$ connected to a Tektronix oscilloscope as in [28]. Two lead zirconate titanate based piezo gauges with nickel electrodes and custom microminiature wiring (Piezo Systems, Inc.) were embedded inside two of the Parylene-C coated particles. The original beads were cut into two parts and the wired piezo elements were glued between the steel parts. The total mass of the assembled beads with sensors was approximately equal to the mass of the original particle $(0.44 \mathrm{~g})$ to preserve the uniformity of the chain. This design ensured a precise calculation of the speed of signal propagation simultaneously with the measurement of the forces acting in the particles. The speed of propagation of the main pulse in the chains was calculated dividing the distance between the sensors by the peak to peak time interval. The accuracy of the speed measurement can be estimated at about $10 \%$ due to the uncertainty in the sensor alignment $( \pm 1 \mathrm{~mm}$ for each sensor).

A third piezo gauge (Kinetic Ceramics, Inc.) was bonded with epoxy on the electrode pads for contacts and reinforced by a $1 \mathrm{~mm}$ brass plate on the top surface. This wall sensor was then placed on the top surface of a long waveguide (vertical steel rod) ending in a steel block to avoid possible reverberations in the system. It was calibrated using the linear momentum conservation law during single impact by a steel ball. Sensors embedded in the particles were calibrated by comparison with the signal from the sensor at the wall under simultaneous impact loading.

To compare the experimentally measured speeds of the solitary waves at different amplitudes with the theoretical prediction derived from the long wave approximation [Eqs. (6) and (7)] we ran a series of experiments at different striker velocities.

\section{RESULTS AND DISCUSSION}

The dynamic behavior of the chains composed of Parylene-C coated beads and steel beads was initially tested in conditions usually resulting in exciting single solitary waves. This is observed when the mass of the striker is close to the mass of one bead [14]. The experimental results corresponding to a chain composed of 21 Parylene-coated beads (4.86 mm diameter, $0.44 \mathrm{~g}$ mass) are shown in Fig. 3(a). It is apparent that under these conditions of loading a single pulse is mainly excited, as expected. In comparison to the bare steel bead case [Fig. 3(b)] the composite particles showed some additional solitarylike small amplitude pulses, following the main pulse, especially noticeable in the first (uppermost) gauge record. Probably this is related to the complex behavior of the coating on the beads composing the system affecting the contact forces between the particles.

In Fig. 3(a) the speed of the main pulse is $197 \mathrm{~m} / \mathrm{s}$, significantly below the level of sound speed in gases at normal conditions. Its duration was about $130 \mu$ s and width equal $\sim 5.3$ particle diameters close to the length of the stationary solution of Eq. (3) based on Hertz interaction law [1] for weakly compressed chains. Similar widths of solitary waves close to five particle diameters were observed at larger amplitudes. This allows us to conclude that the Parylene-C coated bead chain supports the propagation of strongly nonlinear solitary waves with Hertzian exponent 1.5. A higher value of exponent could be expected at larger forces due to the presence of stiffer core which should result in shorter lengths of the solitary wave [14]. Hertz law is derived for linear elastic materials. Nevertheless a Hertzian exponent 1.5 was observed for viscoelastic materials like nylon [10] and PTFE [28].

It is worth noticing that the amplitude of the solitary waves traveling through the chains (especially in the Parylene-C based system) might be heavily affected by the presence of dissipation. Its effects will be addressed in our future research. 

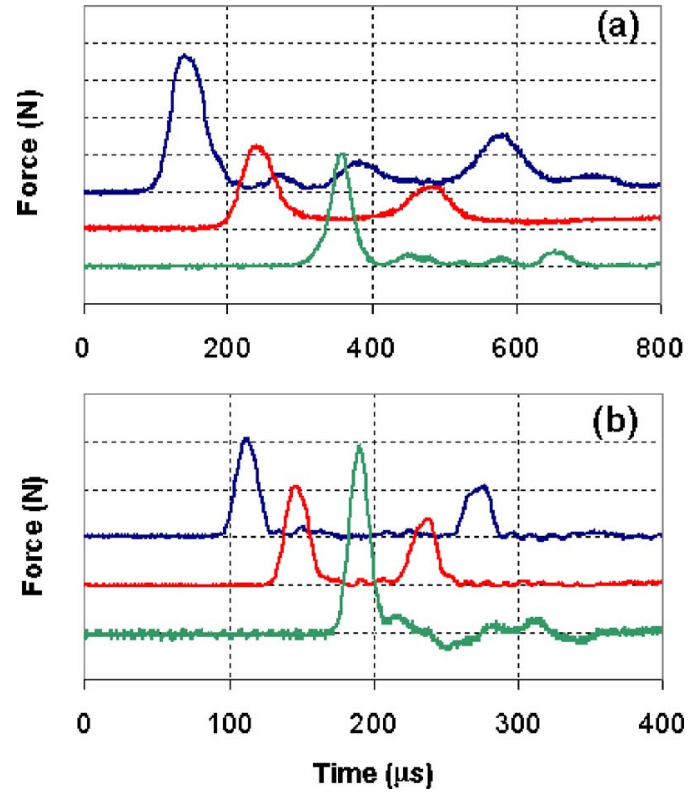

FIG. 3. (Color online) Pulses generated in chains composed of (a) Parylene-C coated steel balls (4.86 mm diameter) and (b) bare stainless steel balls (4.76 mm diameter) for comparison of their amplitudes, speed, width, and attenuation. In both chains sensors are placed in the ninth ball from the bottom (top curves), fifth ball from the bottom (middle curves), and at the bottom wall. The velocity of the striker $\left(0.47 \mathrm{~g} \mathrm{Al}_{2} \mathrm{O}_{3}\right.$ cylinder $)$ was $0.44 \mathrm{~m} / \mathrm{s}$. The scale of the vertical axis is $1 \mathrm{~N}$ for (a) and $5 \mathrm{~N}$ for (b).

It is interesting to compare the behavior of the polymer coated beads with a chain of bare steel beads [Fig. 3(b)] of similar diameter (equal to the diameter of the steel core of the coated beads) and under the same type of loading (same impact striker and same velocity). Steel beads are well known to support strongly nonlinear waves in onedimensional chains [14]. It is evident from comparison between Figs. 3(a) and 3(b) that the behavior of the coated and the not-coated beads differs dramatically in the speed of the propagating pulse $(578 \mathrm{~m} / \mathrm{s}$ for steel beads versus $197 \mathrm{~m} / \mathrm{s}$ for composite beads). Both chains are forming a solitary pulse with width close to five particle diameters. This width corresponds to the length of the stationary solution of Eq. (3) when the pulse's force amplitude is much higher than the initial precompressive force. Reducing the amplitude of the signal traveling through the system to $\sim 0.25 \mathrm{~N}$ we were able to achieve solitary wave speeds in the chain composed of Parylene coated beads down to $\sim 130 \mathrm{~m} / \mathrm{s}$, which is more than two times smaller than the smallest speed of a solitary wave detected for solid nylon beads [10] and is comparable with the one obtained with solid PTFE particles [28]. In principle such media can be designed to support solitary waves with indefinitely small amplitude and speed of propagation. It is reasonable to expect detectable solitary waves with a speed within the order of magnitude of $10 \mathrm{~m} / \mathrm{s}$ or lower, useful, for example, in acoustic delay lines and shock pulse mitigation devices.

It is interesting to follow the dependence of the solitary wave speed on amplitude and compare it with the results from the long wave approximation [Eq. (7)]. The agreement
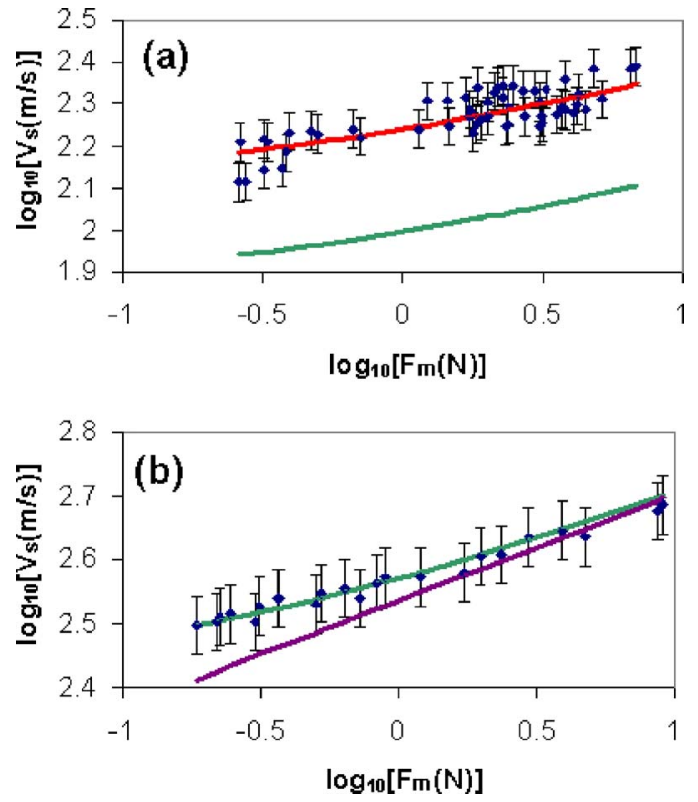

FIG. 4. (Color online) Dependence of the wave speed on the amplitude of the solitary wave in the chains assembled from coated spheres (a) and from stainless steel beads (b). (a) Experimental values (solid dots) and theoretical curves corresponding to the long wave approximation including gravitational preload. The lower curve corresponds to a value of elastic modulus $E=2.76 \mathrm{GPa}$; the upper curve matching the experimental values corresponds to $E$ $=15 \mathrm{GPa}$. (b) Experimental values obtained in a uniform stainless steel chain with the long wave approximation at $E=193 \mathrm{GPa}$ for a sonic vacuum (lower curve) and for a gravitationally precompressed chain (upper curve).

between these approaches was previously demonstrated for strongly nonlinear chains of beads made from different materials $[6,10,28]$.

The experimental results (solid diamonds) and theoretical curves for Parylene are shown in Fig. 4(a), where the bottom curve corresponds to the long wave approximation with elastic modulus provided by the manufacturer, $2.76 \mathrm{GPa}$ [36]. It is evident that there is a large discrepancy between the experimental pulse's speed values and the lower theoretical curve [Eq. (7)]. The experimental data can be described by shifting the theoretical curve up using an increased effective elastic modulus of the composite beads; agreement was obtained with the value $E=15 \mathrm{GPa}$ [see Fig. 4(a), upper curve].

The increased value of the effective elastic modulus of the contact interaction might be due to the high strain, high strain rate deformation of Parylene. This may also be caused by the geometrical constraints induced by the rigid core, because the value of the contact radius is comparable to or larger than the thickness of the coating. The strain rate in the deformed coating was in the interval $10^{2}-10^{3} \mathrm{~s}^{-1}$. It should be mentioned that elastic moduli of polymers are very sensitive to the conditions of testing. For example, measurements of the elastic modulus of Parylene- $\mathrm{C}$ based on the membrane deflection measurements gave a significantly higher value of $4.75 \mathrm{GPa}$ [37] than the one provided by the manufacturer.

In Fig. 4(a) the value of the elastic modulus for the curve matching the experimental data is $\sim 5.4$ times higher than the 
value for Parylene-C provided by the manufacturer. A similar difference was observed also for another polymer based granular material in [28] where the elastic modulus of PTFE appeared to be $\sim 3$ times higher than its nominal value (and matched the Young's modulus extrapolated from the Hugoniot measurements [38]). It is likely that in polymer-based strongly nonlinear systems the effective elastic modulus for the contact interaction might be stress, and strain rate dependent due to the properties of polymers [39].

Further research on the value of the elastic modulus of Parylene-C coating under conditions of dynamic deformation and validity of the Hertz approximation for the contact law of polymer coated beads is necessary to clarify the observed increase of contact stiffness.

Results for the pulse traveling in the bare stainless steel bead chain are presented in Fig. 4(b). In this case the curves represent the long wave approximation at $E=193 \mathrm{GPa}$ for steel in the sonic vacuum (lower curve) and in the gravitationally precompressed case (upper curve) the latter better agreeing with the experimental data. Gravitational precompression was included in Eq. (7) similarly to [33].

In both investigated systems (Parylene coated and steel beads), the theoretical estimate appears to be strikingly close to the experimental results in the whole investigated range, especially taking into account that no adjustable parameter was used in the theoretical consideration for stainless steel beads and a constant effective elastic modulus was used for the contact interaction of the composite particles (though significantly increased in comparison with the manufacturer's value for Parylene).

We may conclude that chains of polymer coated beads with a high density core support the propagation of a strongly nonlinear solitary wave, being another realization of a sonic-vacuum-type system with an exceptionally low speed of signal as observed in chains of beads made from other polymeric materials like Homalite, nylon, or PTFE $[3,4,10,28]$.

Another feature of strongly nonlinear systems without dissipation is the very fast decomposition of a relatively long initial pulse into a train of solitary waves [14]. This type of pulse can be created using a striker with a mass higher than the mass of the particles in the chain $(0.44 \mathrm{~g})$. We used a striker with a mass $m_{s}=1.23 \mathrm{~g}$. Apparently this property may be obscured by the strong dissipation in the polymer-based viscoelastic systems. The number of solitary waves with significant amplitude in which the longer initial pulse is expected to decompose throughout the chain is comparable to the ratio of the striker mass and the mass of the beads in the chain ( $\sim 3$ in this particular experimental setup) [14]. The results of this experiment for a Parylene-C coated beads chain and for a chain made from bare stainless steel beads are shown in Fig. 5.

It is evident that the polymer-based strongly nonlinear chain demonstrates a clear tendency to quickly decompose the initial impulse into a train of three solitary waves on a distance comparable with the soliton width [Fig. 5(a)] as it is also the case for the much less dissipative stainless steel based system [Fig. 5(b)]. In both cases the impulse appears to be almost completely split at the wall, after traveling through only 21 particles, despite the significant difference in
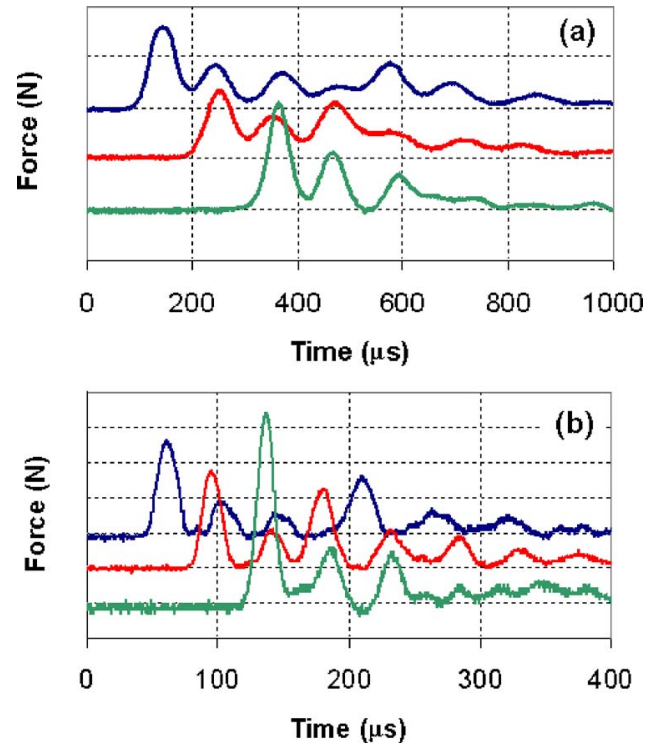

FIG. 5. (Color online) Trains of solitary waves generated in two 21-particle chains composed of (a) Parylene-C coated steel balls $(4.86 \mathrm{~mm}$ diameter) chain and (b) bare stainless steel beads (4.76 mm diameter) chain for comparison. Sensors are placed in the ninth ball from the bottom (top curve), fifth ball from the bottom (middle curve), and at the bottom wall. The velocity of striker (1.23 $\mathrm{g} \mathrm{Al}_{2} \mathrm{O}_{3}$ cylinder) was $0.44 \mathrm{~m} / \mathrm{s} . Y$-axis scale is $1 \mathrm{~N}$ for (a) and $5 \mathrm{~N}$ for (b).

pulse speed. Previous experimental work with chains of steel, glass, brass, Homalite, Nylon, and PTFE beads $[1,3,4,6,10,28,32]$ validated the prediction of the existence of strongly nonlinear solitary waves as stationary solutions of the wave equation [Eq. (3)]. At the same time, it is evident that the bare stainless steel chain supports a more pronounced splitting of the initial pulses into a train of solitary waves. This is most probably related to the higher dissipation in the contact area between the Parylene coated particles. The presence of increased dissipation in the coated beads is evident from the faster decay of the pulses and the larger overlap of the peaks in the train. The presence of an increased viscous dissipation might contribute to a delay in the signal splitting, as observed in [40] for a chain of particles immersed in liquids of different viscosities (oil and glycerol).

It is important to investigate the influence of the particle size on the system's behavior for application purposes. Reducing the diameter of the Parylene- $\mathrm{C}$ coated beads may result in different stresses and strain rate conditions at the contact area, affecting the behavior of the system; specifically, increasing the dissipation, as it was observed in a chain of PTFE beads [28]. We conducted experiments with smaller diameter steel balls $(2.38 \mathrm{~mm})$ coated by a $50 \mu \mathrm{m}$ Parylene-C layer (total bead diameter is $2.48 \mathrm{~mm}$ ). Experimental results are presented in Fig. 6. The sensor in this particular setup was placed only at the wall for simplicity. Impacts were generated using a 2-mm-diameter steel ball [0.036 g, Fig. 6(a)] and a 4.76-mm-diameter PTFE ball $[0.123 \mathrm{~g}$, Fig. 6(b)] at velocity $0.89 \mathrm{~m} / \mathrm{s}$ with no precompression acting on the chains (other than the practically negligible gravitational loading due to vertical setup). It appears 

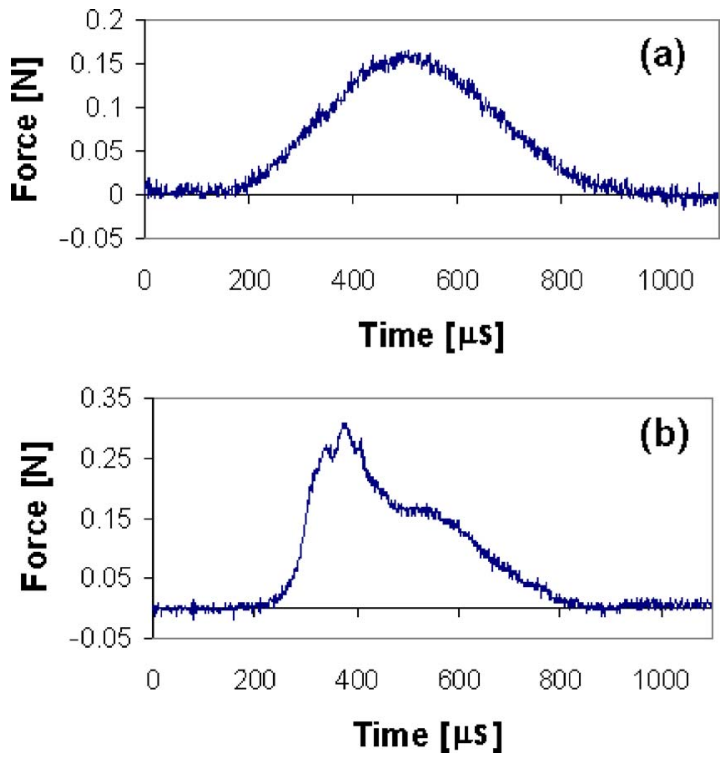

FIG. 6. (Color online) Solitarylike wave (a) and the shocklike nonstationary pulse (b) detected on the wall supporting a chain of 22 smaller Parylene-C balls ( $2.48 \mathrm{~mm}$ diameter, $m=0.04 \mathrm{~g}$ ). (a) Impact at velocity $0.89 \mathrm{~m} / \mathrm{s}$ with 2-mm-diameter steel ball $(0.036 \mathrm{~g})$; (b) impact at velocity $0.89 \mathrm{~m} / \mathrm{s}$ with a $4.76-\mathrm{mm}$-diameter PTFE ball with larger mass $0.123 \mathrm{~g}$.

that the smaller diameter Parylene-C coated particles tend to form a single solitarylike wave when impacted by a striker of the same mass as a bead [Fig. 6(a)]. This is a typical feature of the sonic-vacuum-type behavior [14]. When impacted by a striker with higher mass the effect of dissipation appears to delay the solitary wave splitting [Fig. 6(b)].

Parylene can be plastically deformed even at room temperature [41]; this restricts the amplitude of the pulses that can be treated based on the elastic approach. Cracking of the coating layer was seldom observed at higher dynamic force amplitude ranges $(\geqslant 3 \mathrm{~N})$. Despite this, we observed a consistent behavior in terms of the wave speed dependence on amplitude [Fig. 4(a)]. Typical crack morphology is depicted in Fig. 7. The development of median cracks before the Hertzian cracks is evident. We explain this phenomenon in analogy to the behavior of polymers under spherical indentation [41]. Finite element studies demonstrated that under significant displacements in the contact area the polymeric material is pushed outward, causing hoop tensile stresses just outside the contact zone. In our experiments, the median cracks ap-

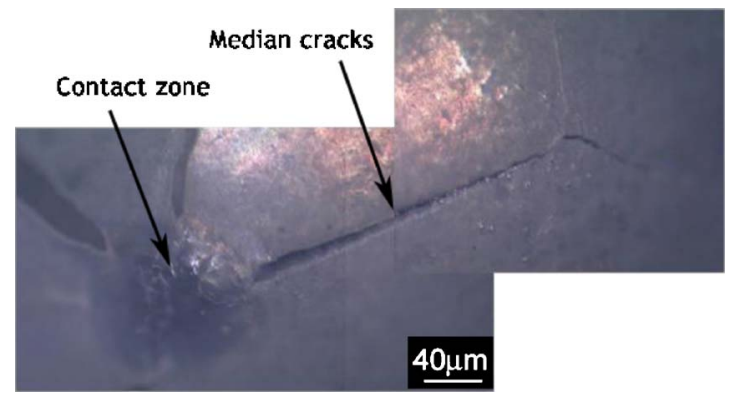

FIG. 7. Four typical median cracks originating away from the deformed contact zone (related to the area with radius $b$ depicted in Fig. 1) observed after high amplitude dynamic testing with a maximum force on the level of $\sim 3 \mathrm{~N}$. Note that they appear before the Hertzian ring cracks which are supposed to originate on the outside area of the contact zone.

peared prior to any ring cracks around the contact area, in agreement with the numerical results in [41]. We may expect that under very large contact forces and high strains in the contact area, the steel cores of neighboring beads might come into direct contact, increasing the stiffness of the system and therefore abruptly increasing the speed of signal propagation.

\section{CONCLUSION}

Chains composed of steel beads of different diameters coated by a $50 \mu \mathrm{m}$ layer of Parylene-C were assembled and investigated under different conditions of impact loading. It was demonstrated that chains of composite particles with a "hard" core and a "soft" interacting contact do support the Hertzian type of interaction and the formation of single and trains of strongly nonlinear solitary waves. The soft interacting coating enabled a low signal propagation speed due to the low elastic modulus of the contact interaction and supported the formation of strongly nonlinear solitary waves despite its viscoelastic nature. The formed solitary waves had a speed of propagation well below sound speed in air. Decomposition of the incoming signal into trains of solitary waves was observed only for the larger diameter beads. At relatively high amplitudes of solitary waves we observed the formation of median cracks prior to the formation of ring Hertzian cracks.

\section{ACKNOWLEDGMENT}

This work was supported by NSF (Grant No. DCMS03013220).
[1] V. F. Nesterenko, Prikl. Mekh. Tekh. Fiz. 24, 136 (1983) [J. Appl. Mech. Tech. Phys. 24, 733 (1984)].

[2] A. N. Lazaridi and V. F. Nesterenko, Prikl. Mekh. Tekh. Fiz. 26, 115 (1985) [J. Appl. Mech. Tech. Phys. 26, 405 (1985)].

[3] A. Shukla, M. H. Sadd, Y. Xu, and Q. M. Tai, J. Mech. Phys. Solids 41, 1795 (1993).

[4] Y. Zhu, F. Sienkiewicz, A. Shukla, and M. Sadd, J. Eng. Mech. 10, 1050 (1997).
[5] V. F. Nesterenko, J. Phys. IV 4, C8-729 (1994).

[6] C. Coste, E. Falcon, and S. Fauve, Phys. Rev. E 56, 6104 (1997).

[7] M. Manciu, S. Sen, and A. J. Hurd, Physica A 274, 607 (1999).

[8] M. Remoissenet, Waves Called Solitons (Concepts and Experiments), 3rd ed. (Springer-Verlag, Berlin, 1999).

[9] E. J. Hinch and S. Saint-Jean, Proc. R. Soc. London, Ser. A 
455, 3201 (1999).

[10] C. Coste and B. Gilles, Eur. Phys. J. B 7, 155 (1999).

[11] J. Hong, J.-Y. Ji, and H. Kim, Phys. Rev. Lett. 82, 3058 (1999).

[12] A. Chatterjee, Phys. Rev. E 59, 5912 (1999).

[13] E. Hascoet and H. J. Herrmann, Eur. Phys. J. B 14, 183 (2000).

[14] V. F. Nesterenko, Dynamics of Heterogeneous Materials (Springer-Verlag, New York, 2001), Chap. 1.

[15] S. Sen, M. Manciu, R. S. Sinkovits, and A. J. Hurd, Granular Matter 3, 33 (2001).

[16] M. Manciu, S. Sen, and A. J. Hurd, Physica D 157, 226 (2001)

[17] M. Manciu, S. Sen, and A. J. Hurd, Phys. Rev. E 63, 016614 (2001).

[18] J. Hong and A. Xu, Phys. Rev. E 63, 061310 (2001).

[19] S. Sen and M. Manciu, Phys. Rev. E 64, 056605 (2001).

[20] F. S. Manciu and S. Sen, Phys. Rev. E 66, 016616 (2002).

[21] C. A. Arancibia-Bulnes and J. C. Ruiz-Suarez, Physica D 168, 159 (2002).

[22] A. Rosas and K. Lindenberg, Phys. Rev. E 68, 041304 (2003).

[23] R. Reigada, A. Samiento, and K. Lindenberg, Chaos 13, 646 (2003).

[24] S. Sen, S. Chakravarti, D. P. Visco, Jr., D. T. Wu, M. Nakagawa, and J. H. Agui, Jr., in Impulse Propagation in Granular Systems, edited by V. M. Kenkre and K. Lindenberg, AIP Conf. Proc. No. 658 (AIP, Melville, NY, 2003), pp. 357-379.

[25] A. Rosas and K. Lindenberg, Phys. Rev. E 69, 037601 (2004).

[26] A. I. Musienko and L. I. Manevich, Phys. Usp. 47, 797 (2004).
[27] M. Kastner, Phys. Rev. Lett. 92, 104301 (2004).

[28] C. Daraio, V. Nesterenko, E. Herbold, and S. Jin, Phys. Rev. E 72, 016603 (2005).

[29] C. Goldenberg and I. Goldhirsch, Nature (London) 435, 188 (2005).

[30] S. Luding, Nature (London) 435, 159 (2005).

[31] J. Hong, Phys. Rev. Lett. 94, 108001 (2005).

[32] S. Job, F. Melo, A. Sokolow, and S. Sen, Phys. Rev. Lett. 94, 178002 (2005).

[33] C. Daraio, V. F. Nesterenko, E. B. Herbold, and S. Jin, e-print cond-mat/0506513, Phys. Rev. E. (to be published).

[34] V. F. Nesterenko, C. Daraio, E. B. Herbold, and S. Jin, Phys. Rev. Lett. 95, 158702 (2005).

[35] H. Hertz and J. Reine, Angew. Math. 92, 156 (1881).

[36] AcraBall Manufactoring Co. Product catalog.

[37] C. S. Shih, T. A. Harder, and Y. C. Tai, Microsyst. Technol. 10, 407 (2004).

[38] W. J. Carter and S. P. Marsh, Los Alamos Report No. LA13006-MS, 1995 (unpublished).

[39] F. J. Zerilli and R. W. Armstrong, in Shock Compression of Condensed Matter-2001, edited by M. D. Furnish, N. N. Thadhani, and Y. Horie, AIP Conf. Proc. No. 620 (AIP, Melville, NY, 2002), pp. 657-660.

[40] E. B. Herbold, V. F. Nesterenko, and C. Daraio, in Shock Compression of Condensed Matter -2005, edited by M. D. Furnish and M. Elert (to be published); e-print cond-mat/0512367.

[41] K. Sadeghipour, W. Chen, and G. Baran, J. Phys. D 27, 1300 (1994). 